\title{
Segmentation of 3D Mesh Models Based on Conformal Factor
}

\author{
Yang Jun ${ }^{1 *}$, Wang Maozheng ${ }^{2}$ and Yan $\operatorname{Han}^{1}$ \\ ${ }^{1}$ School of Electronic and Information Engineering, Lanzhou Jiaotong University, \\ Lanzhou 730070, China \\ ${ }^{2}$ School of Automation and Electrical Engineering, Lanzhou Jiaotong University, \\ Lanzhou 730070, China \\ I*yangj@mail.lzjtu.cn
}

\begin{abstract}
The segmentation technique of $3 D$ mesh models plays a key role in comprehending and processing digital geometric models. A new segmentation algorithm for $3 D$ mesh models based on conformal factor and k-means clustering is proposed to deal with the existing problems in segmentation methods, which includes a lack of semantic information and low performance in segmentation results. It is easy to obtain an effective segmentation results with semantic information since the conformal factors carry global feature information of the model. Firstly, the discrete Gaussian curvatures of each vertex are figured out. Secondly, the conformal factors are calculated by employing LaplaceBeltrami operators. Finally, the clustering of meshes is realized by modified k-means clustering algorithm. Experimental results show that the proposed algorithm not only can achieve meaningful segmentation of $3 D$ models, but also has good anti-jamming performance caused by pose variation of the model.
\end{abstract}

Keywords: mesh segmentation; conformal factor; Gaussian curvature; LaplaceBeltrami operator; $k$-means clustering

\section{Introduction}

With the rapid development of 3D scanning technology, the related techniques of the geometrical processing of 3D model have attracted considerable concern. Since the original triangular mesh model lacks sufficient structure characteristics and semantic information, the understanding of 3D geometrical model and relevant geometric processing is greatly hindered. Therefore, it is important for model understanding to partition the mesh model into meaningful components or surfaces in accordance with certain rules. Segmentation method of the mesh model plays a critical role in geometric modeling and digital geometry processing. It is widely used in fields like surface simplification and segmentation [1], geometric compression and transmission [2], texture mapping [3], animation and geometric deformation [4], model retrieval [5], local parameterization [6], and reverse engineering [7]. Zuckerberger et al., [5] decompose polyhedral surface into a certain number of meaningful segments and utilize every patch as a basic shape. Then, the neighboring relationships between patches are identified, property graph of the mesh surface model is constructed, and similar property graph is matched by searching to accomplish the purpose of retrieval. However, in the process of model segmentation, different selections of feature descriptor lead to different segmentation results, influencing the results of model matching. Hence, selection of feature descriptor of the mesh model is extremely important for mesh segmentation.

In this paper, a descriptor, i.e., conformal factor, with characteristic information of the $3 \mathrm{D}$ mesh model is proposed based on the conformal mapping. Conformal factor is a global feature descriptor, with the advantages of invariance in non-rigid isometric 
transformation and easy to calculate. So, to some extent, the influence of model posture variation on the result of segmentation is overcome.

\section{Related Work}

In recent years, extensive attention has been focused on segmentation of the 3D model. Lots of segmentation algorithms have been presented and some critical problems in model segmentation have been solved. These methods can be classified into two categories: mesh segmentation based on local geometric characteristics and topology information of the model and meaningful parts segmentation based on semantic information. For the former, there are Reeb Graph, central axis, Shock Graph, and skeleton extraction, etc. Mesh segmentation partitions the model into a certain number of surfaces, such as planar surface, sphere surface, and polynomial surface. 3D mesh model is partitioned in literature [8] utilizing local geometric properties such as edge curvature, face curvature, and vertex normal vector. The result can show simple concave-convex information of each area and the method is suitable for dense mesh model, but meaningful segmentation of the model is failed to achieve. Xiao et al. [9] proposes a segmentation algorithm based on Discrete Reeb Graph (DRG) and it partitions 3D points cloud data of the human body. It is a topological approach that realizes partition of the model by detecting critical nodes in the DRG and building topology branches of all parts of the human body. However, over-segmentation problem is easy to exist because the approach is intrinsically not qualified to handle postures where limbs are joined together, for example closed legs, or arm touching the torso. Moreover, for different models or different postures of the same model, different Reeb Graphs are required to construct and different Morse function are required to choose, resulting in poor adaptability to other models. A 3D skeleton algorithm based on level-progressive is presented in reference [10]. Firstly, the prominent feature faces of the 3D model based on multi-dimensional scaling (MDS) are calculated. Secondly, feature faces are used as the seed points and k-ring strip growing algorithm is used to compute the center of each level and connect these centers to construct skeleton line of each segmentation block, which is then connected to obtain whole skeleton of the model. The skeleton of the 3D model extracted by the algorithm is rather smooth, but segmentation problem of multi-connected model is not considered. Thus, the algorithm is only applied to single connected and mesh evenly distributed model with ring topology characteristics.

Meaningful segmentation algorithm based on semantic information is realized usually by combining with clustering algorithm. Currently, most of the clustering algorithms used in mesh model segmentation are inspired by image segmentation methods, namely, 2D image segmentation algorithms are applied to 3D model. For example, watershed algorithm, region growing algorithm, iterative clustering algorithm, hierarchical decomposing and merging algorithm, spectral clustering algorithm, etc. Watershed method in image processing used to segment 3D surface meshes is first described in literature [11]. The segmentation is accomplished through calculating the total curvature of the mesh surfaces, and then watershed algorithm is used to partition the surface into patches. However, this method is sensitive to segmentation threshold, so oversegmentation exists when threshold is improperly selected. Koschan et al., [12] propose a Fast Marching Watersheds algorithm that segments a triangle mesh into visual parts based on human vision theory. Their implementation computes the principal curvatures and principal directions at each vertex of a mesh, and then hill-climbing watershed algorithm identifies regions bounded by contours of negative curvature minima. The approach improves the speed of mesh partition and reduces over-segmentation to a certain extent, but the segmentation boundaries are not smooth enough. Zhang et al., [13] present a simple and efficient algorithm for part decomposition of compound objects based on Gaussian curvature analysis. Vertex curvature of the meshes is computed, then specified 
curvature threshold is used to mark vertexes with large negative curvature as boundary points and the remaining vertexes are labeled as seed points. Starting from these seed points, growth rule is used as zone marker until boundary points are met. Although the algorithm can obtain segmented patches with clean boundaries and homogeneous curvature, it is only suitable for the segmentation of simple mechanical CAD model. Liu et al., [14] put forward spectral clustering to 3D mesh segmentation based on spectrum analysis theory. Given a set of mesh faces, an affinity matrix which encodes the likelihood of each pair of faces belonging to the same group is first constructed. Spectral methods then use selected eigenvectors of the affinity matrix or its closely related group Laplacian to obtain data representations that can be more easily clustered. This algorithm favors segmentation along concave regions, which is inspired by human perception, and can achieve high-quality segmentation results on 3D meshes. However, this algorithm cannot guarantee to find the best boundary, especially when a greater portion of this boundary lies in featureless regions. In addition, the algorithm cannot guarantee smooth segmentation boundary.

3D mesh segmentation is mainly through extracting characteristics of the model and selecting appropriate clustering algorithm to partition model into meaningful components or surfaces, so that relevant geometric processing can be easily done. Therefore, the extraction of feature descriptor is particularly important. The most commonly used feature descriptors in mesh segmentation algorithm are: Gaussian Curvature (GC), Geodesic Distance (GD), Shape Diameter Function (SDF), Average Geodesic Distance (AGD), Shape Contexts (SC), Conformal Factor (CF), etc. Among them, SC and CF represent global feature information of the model, while the others represent local feature information of the model.

Currently, the major problems in mesh segmentation algorithm are: over segmentation, unsmooth segmentation boundary, poor robustness against model posture variation, low performance, etc. Although, some latest algorithms overcome the over segmentation problem, some other problems still exist: First, inappropriate selection of the feature descriptor. For example, local feature descriptors like curvature and geodesic distance are sensitive to posture variation, which is easy to cause incorrect segmentation. Second, for realizing spectral embedding, geodesic distance is used to construct mesh model and affinity matrix, leading to large amount of calculation and low segmentation efficiency.

A 3D mesh segmentation algorithm based on conformal factor is proposed in this paper. The discrete Gaussian curvatures of each vertex are obtained. To accomplish the extraction of mesh feature, conformal factors of each vertex of the mesh model are calculated using Laplace-Beltrami operators. Then, a modified k-means clustering algorithm is implemented to achieve segmentation of the mesh model. Conformal factor can present global feature information of the mesh model in low dimensional space and it carries certain semantic information, resulting in meaningful components segmentation and high segmentation efficiency.

\section{Gaussian Curvature and Laplace-Beltrami Operator}

\subsection{Gaussian Curvature}

Gaussian curvature of vertexes of the 3D mesh model can directly reflect local concavo-convex information of the model. When Gaussian curvature is positive, the vertex is on the convex and the larger the Gaussian curvature, the sharper the convex will be. On the contrary, when Gaussian curvature is negative, the vertex is on the concave, and the smaller the Gaussian curvature, the deeper the concave will be. Furthermore, difference in Gaussian curvature among adjacent points can measure the extent of changes of concave and convex surface near the point.

Let triangle mesh model be $\mathbf{M}$, and assume sets $\mathbf{V}, \mathbf{F}$, and $\mathbf{E}$ to be vertex, line, and face 
of the meshes, respectively. The discrete Gaussian curvature of any vertex of the mesh model is defined as:

$K_{v}=3\left(2 \pi-\sum_{f \in \mathbf{F}_{v}} \alpha_{v}^{f}\right) / \sum_{i=1}^{n} S_{i}$

Where, $\mathbf{F}_{v}$ is the surfaces with common vertex $v$ in set $\mathbf{F} ; S_{i}$ is the area of triangle formed by vertex $v$ and its adjacent points, and $S_{i}$ can be calculated by:

$S_{i}=\left|\overrightarrow{e_{i}} \times \overrightarrow{e_{i+1}}\right| / 2$

$\alpha_{v}^{f}$ is vertex degrees of triangle fi formed by vertex $\mathrm{v}$ and its adjacent points of first layer, the edges of triangle across point $\mathrm{v}$ are $\overrightarrow{e_{i}}=\overrightarrow{v v_{i}}$ and $\overrightarrow{e_{i+1}}=\overrightarrow{v v_{i+1}}$. So, $\alpha_{v}^{f}$ can be obtained by:

$$
\alpha_{v}^{f_{i}}=\arccos \left[\frac{\overrightarrow{e_{i}} \bullet \overrightarrow{e_{i+1}}}{\left|\overrightarrow{e_{i}}\right| \cdot\left|\overrightarrow{e_{i+1}}\right|}\right]
$$

The parameters needed in calculation of discrete Gaussian curvature of the 3D mesh model are shown in Figure 1.

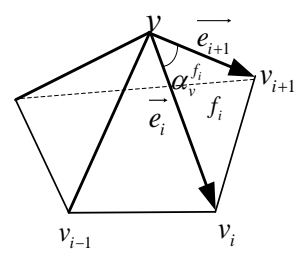

Figure 1. Parametric Sketch of Calculation of Gaussian Curvature

\subsection{Laplace-Beltrami Operator}

Let $\Psi$ be a $C^{2}$ real-valued function defined on a differentiable manifold $\mathbf{M}$ with Riemannian metric. The Laplace-Beltrami operator $\Delta$ is defined as the divergence of the gradient [15]:

$\Delta(\Psi)=\operatorname{div}(\operatorname{grad}(\Psi))$

where grad and div are the gradient and divergence on the manifold $\mathbf{M}$.

Let $\mathbf{G}=(\mathbf{V}, \mathbf{E})$ be the graph of triangulation of the Riemannian manifold. Consider the scalar function $h: \mathbf{V} \rightarrow \square$ is the restriction of $\Psi$ to $\mathbf{V}$. Assume $\mathbf{W}$ be an oriented incidence matrix corresponding to mesh graph $\mathbf{G}$. The $\mathbf{W} \in \square^{\mathrm{nxm}}$ is a vertex-edge incidence matrix defined as:

$\mathbf{W}_{i e}=\left\{\begin{array}{l}-1, \text { if } i \text { is the inital vertex of edge } e \\ +1, \text { if } i \text { is the terminal vertex of edge } e\end{array}\right.$

Then, the operator $\mathbf{W}^{\mathrm{T}} h: \mathbf{E} \rightarrow \square$ acts on the set $\mathbf{E}$ of oriented edges, and $|\mathbf{E}|=2|\mathbf{E}|$. The operator can be expressed as:

$\left(\mathbf{W}^{\mathrm{T}} h\right)(\mathrm{e})=h\left(e^{+}\right)-h\left(e^{-}\right)$

where $e^{-}$and $e+$ are the terminal and initial vertexes of edge $e$, respectively. 
Thus, the aforementioned operators can be approximately considered as the gradient $\Psi$ of at the edge $e$. These operators follow the relationship $\mathbf{Q}=\mathbf{W} \mathbf{W}^{\mathrm{T}}$ that provides an analogue of the divergence of the gradient, giving a combinatorial version of the LaplaceBeltrami operator. When the triangular mesh graph $\mathbf{G}$ becomes dense enough, the restriction of Laplace-Beltrami operator $\Delta(\Psi)$ to vertex $\mathbf{V}$ is expected to be close to operator $\mathbf{Q} h$.

\section{Conformal Factor}

The uniformization theorem [16] states that any 2D manifold surface can be conformally mapped to a surface with the same topology having constant Gaussian curvature. Such a mapping can be achieved by defining a positive scalar function on the surface, and this function is used locally to change the measurement of the surface (which can be considered as infinitesimal patches of the scaling surface). The scaling function $\varphi$ (also known as the conformal factor) only depends on the Gaussian curvature of the surface. Hence, it is invariant to isometric transformations. In the continuous set, conformal factor $\varphi$ is defined as the solution of non-linear partial differential equation:

$\nabla^{2} \varphi=\mathbf{K}^{\text {orig }}-\exp (2 \varphi) \mathbf{K}^{\text {targ }}$

where $\mathbf{K}^{\text {orig }}$ is Gaussian curvature of the surface, and $\mathbf{K}^{\text {targ }}$ is the target value of Gaussian curvature.

According to the conformal mapping theory, Ben-Chen et al., [17] present a new descriptor, i.e., conformal factor, which reflects the global characteristics of the discrete mesh model and is invariant under non-rigid quasi-isometric transformations. Conformal factor $\varphi$ is the solution of sparse linear equations:

$$
\mathbf{L} \varphi=\mathbf{K}^{\text {targ }}-\mathbf{K}^{\text {orig }}
$$

where $\mathbf{L}$ is the discrete Laplace-Beltrami operator with cotangent weight.

The target Gaussian curvature of a vertex $v$ in the mesh is uniform Gaussian curvature, which can be expressed as:

$$
\mathbf{K}_{v}^{\text {targ }}=\left(\sum_{i \in \mathbf{V}} \mathbf{K}_{i}^{\text {orig }}\right) \frac{\sum_{f \in \mathbf{F}_{v}} \frac{1}{3} \operatorname{area}(f)}{\sum_{f \in \mathbf{F}} \operatorname{area}(f)}
$$

where $\mathbf{F}_{v}$ is the set of faces in $\mathbf{F}$ which share the vertex $v$, and area $(f)$ is the area of face $f$.

For a connected mesh, the Laplace operator has co-rank 1. Therefore, the solution of conformal factor $\varphi$ is only depends on an additive constant, and it has zero mean. Since the Laplace operator is sparse and symmetric, equation (8) can be used to obtain conformal factor $\varphi$ efficiently.

\section{Modified $k$-means Clustering Algorithm}

$k$-means clustering algorithm is an iterative redistribution clustering algorithm based on square error. The algorithm selects $k$ initial clustering center, and assigns every object to the nearest cluster point to obtain a group of cluster. Then, the current clustering center is calculated to be the new cluster point, and whether the clustering center has changed will be determined. If the clustering center has changed, use the new clustering center to substitute the old clustering center, and every data in the sample will be traversed and assigned to the new clustering center. If the clustering center has not changed, it means clustering average error criterion function (where $\Gamma_{j}$ is the $j^{\text {th }}$ cluster sample set, $y$ is the 
sample in cluster, $m_{j}$ is the average value of the $j^{\text {th }}$ cluster) converges to a constant value, in this case, the algorithm will be terminated and the clustering results will be obtained.

The key to the $k$-means clustering algorithm is the selection of the initial clustering center. If the initial clustering center is properly selected, the clustering algorithm will have satisfactory results and efficient execution. The initial clustering center can be either randomly selected, or specified. In this part, it is selected based on geometrical characteristics and Gaussian curvature of the mesh model: 1) compute Gaussian curvature of each vertex of the mesh model, sort the results, and select the largest negative curvature as the first initial clustering center; 2) use this clustering center as the center and threshold value as the radius to form a circle; 3 ) set the largest negative curvature among the remaining points in this circle as the next clustering center; 4) repeat the above steps until $k$ initial clustering center meet the cluster.

\section{Experimental Results and Discussion}

Conformal factor can clearly denote global characteristic distribution and part of the semantic information of the model, which is conducive to meaningful segmentation of the components. Feature distribution of several 3D mesh models is depicted in Figure 2, where the parts with same color mean the same feature, and vice versa. Areas with different colors represent different values of conformal factors of vertexes in these areas, for example, deep red means the largest value of conformal factor, while deep blue means the smallest value of conformal factor.

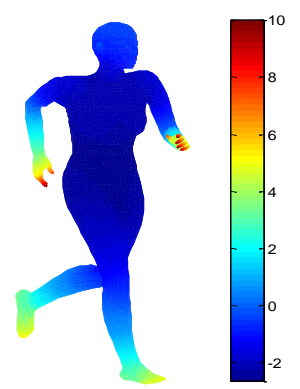

(a) Men

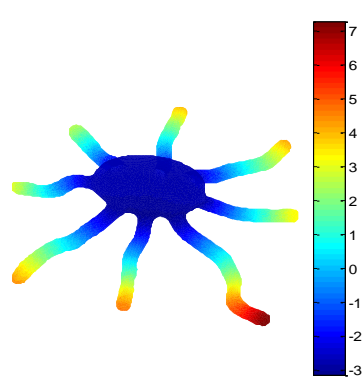

(b) Octopus

Figure 2. Distribution of Feature Descriptor(conformal factor)

Compared with the segmentation result of spectral clustering algorithm [14], which is shown in Figure 3, the proposed algorithm can obtain more accurate segmentation result, can basically achieve segmentation based on components, and can completely differentiate frame of the glasses from legs of the glasses. However, in reference [14], frame and legs of the glasses are partitioned into the same component, and the left and right side of the glasses are considered as two different parts.

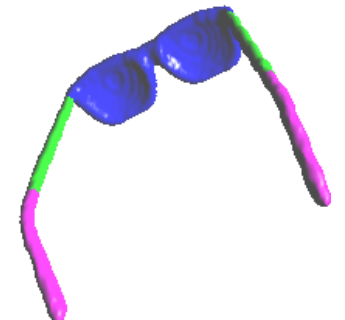

(a) Algorithm in this paper

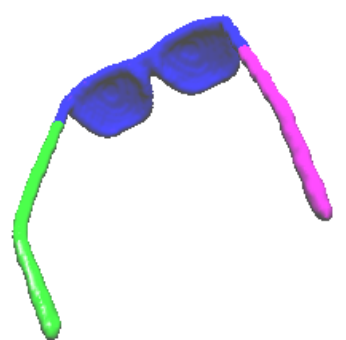

(b) Algorithm in reference[14]

Figure 3. Comparison of segmentation of different algorithms 
Compared with the segmentation result of mesh segmentation algorithm based on edge curvature and local characteristics [18], which is shown in Figure 4, the proposed algorithm can completely and quickly partition the CAD model into meaningful parts and can solve the over-segmentation problem.

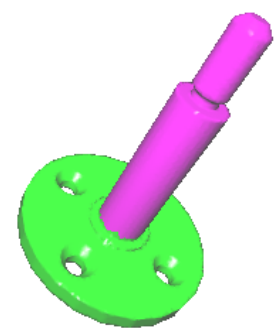

(a) Algorithm in this paper

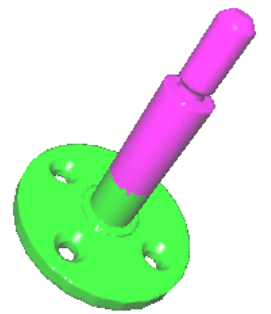

(b) Algorithm in reference[18]

Figure 4. Comparison of segmentation of different algorithms

As for segmentation efficiency, the proposed algorithm has no need to construct affinity matrix, leading to greatly reduced time in computing feature descriptor and improved segmentation efficiency. However, literature [19] needs to reduce its dimensionality of the data when clustering. This is time consuming, and it will bring negative influence on the segmentation result if the dimensionality is improper selected. Conformal factor describes global characteristics of the mesh model in low dimensional space (1D) in the proposed algorithm, thus, the clustering efficiency is improved. Comparison of performance of different algorithms is shown in table 1.

Table 1. Comparison of performance of different algorithms

\begin{tabular}{|c|c|c|c|c|}
\hline \multirow[b]{2}{*}{$\begin{array}{c}\text { Mesh model } \\
\text { (Vertex number ) }\end{array}$} & \multicolumn{2}{|c|}{ Algorithm in this paper } & \multicolumn{2}{|c|}{ Algorithm in reference[19] } \\
\hline & $\begin{array}{c}\text { Feature extraction } \\
\text { time }(\mathrm{s})\end{array}$ & $\begin{array}{l}\text { Cluster time } \\
\text { (s) }\end{array}$ & $\begin{array}{c}\text { Feature extraction } \\
\text { time(s) }\end{array}$ & $\begin{array}{l}\text { Cluster time } \\
\text { (s) }\end{array}$ \\
\hline Hand(7500) & 7.263 & 0.001 & 14.312 & 0.004 \\
\hline Octopus (9200) & 9.258 & 0.001 & 22.513 & 0.006 \\
\hline Glass(12194) & 13.274 & 0.002 & 21.825 & 0.009 \\
\hline Cup(15006) & 15.924 & 0.003 & 24.537 & 0.011 \\
\hline
\end{tabular}

It can be observed from Table 1 that with the increase of vertexes of the mesh model, the consumption of time increases (both in this paper and in reference [19]) during the process of feature calculation and clustering. However, the proposed algorithm still takes significantly less time than reference [19], in other words, the segmentation efficiency of $3 \mathrm{D}$ mesh model is improved in the proposed algorithm.

The experimental results in Figure 5 and Figure 6 show that the proposed algorithm has good robustness against posture variation when we partition octopus model and hand model with different postures. Since the proposed algorithm uses a combination of vertex Gaussian curvature and Laplace-Beltrami operator to calculate conformal factor, the results not only have isometric invariance of the vertex Gaussian curvature, but also carry global characteristics information of 3D model, and also improve representation of the discrete Gaussian curvature for concave-convex information. Therefore, as long as the topology remains unchanged during the process of posture variation, the solving of conformal factor of the meshes can be conformal mapped to a surface with constant Gaussian curvature. As a result, meaningful segmentation with higher precision can be realized.

Several types of 3D model are partitioned and meaningful segmentation is obtained using the proposed algorithm, as shown in Figure 7. The segmentation results indicate that the proposed algorithm has a strong feasibility. 


\section{Conclusion}

This paper presents a segmentation algorithm based on conformal factor and k-means cluster for 3D mesh model. The proposed algorithm improves segmentation efficiency by eliminating the need to construct affinity matrix and improving the calculation speed of feature descriptor. It also has good robustness against posture variation of mesh models. The experimental results show that compared with existing algorithms the proposed algorithm can achieve fast meaningful segmentation and significantly improved segmentation results. However, the segmentation boundary is not smooth enough in the proposed algorithm. The realization of higher level of semantic segmentation and optimization of the smooth segmentation boundary will be explored in the later work.

\section{Acknowledgments}

The authors would like to thank the reviewers for their valuable comments. This work is supported by Natural Science Foundation of China under grant No.61462059, China Postdoctoral Science Foundation Funded Project under grant No. 2013M542396, and Fundamental Research Funds for Gansu Provincial Universities under grant No. 214142.
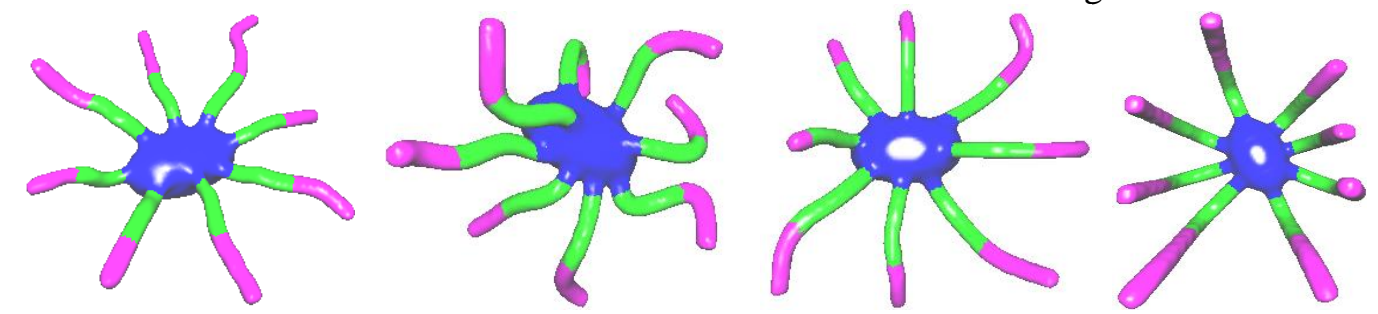

Figure 5. Consistent Segmentation Results of Octopus Model with Different Poses
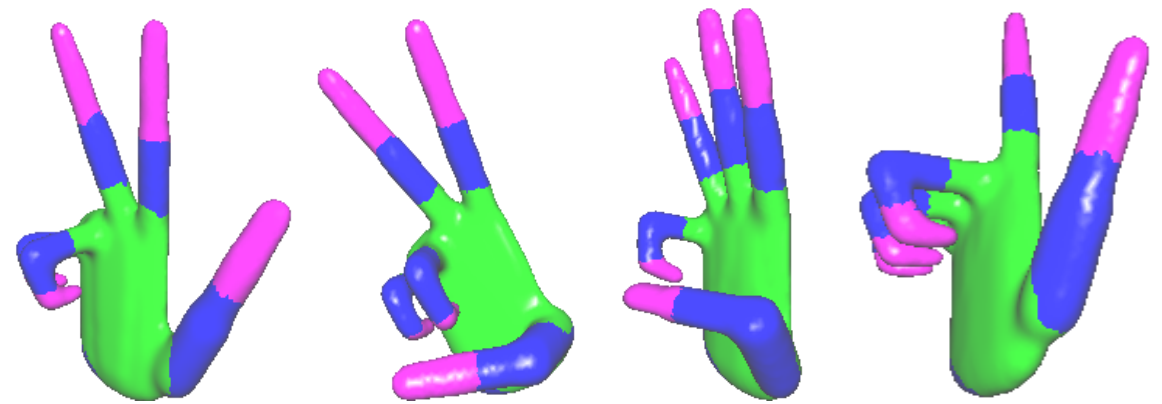

Figure 6. Consistent Segmentation Results of Hand Model with Different Poses
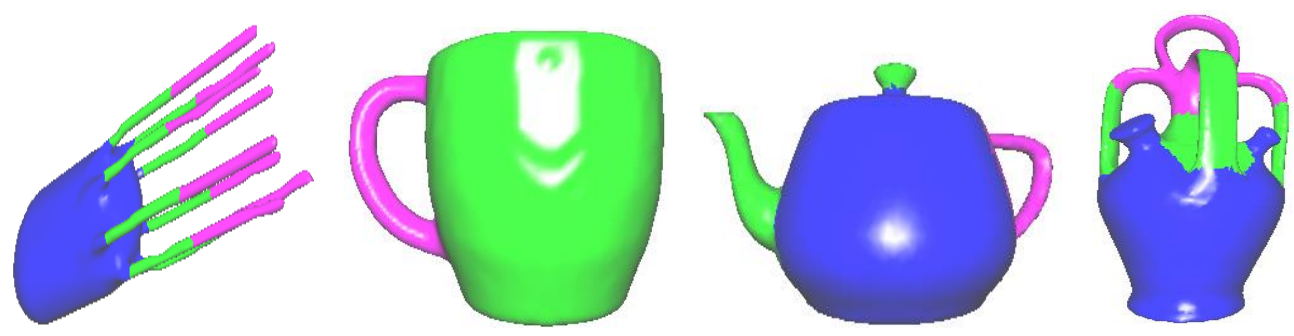

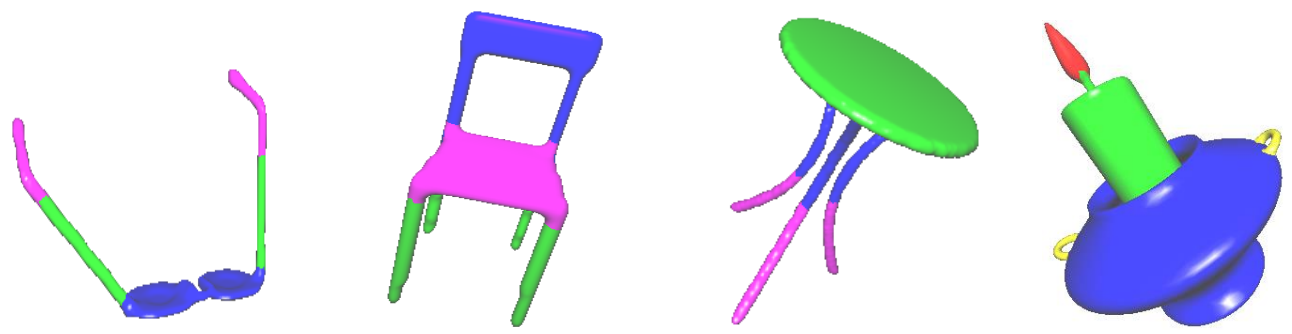

\section{Figure 7. Segmentation Results of other Models}

\section{References}

[1] M. Garland, A. Willmott, P. Heckbert, "Hierarchical face clustering on polygonal surfaces", Proceedings of ACM Symposium on Interactive 3D Graphics, North Carolina, USA, (2001), pp. 49-58.

[2] G. DerK, R. W. Sumner, "Inverse kinematics for reduced deformable models", ACM Transactions on Graphics, vol. 25, no. 3, (2006), pp. 1174-1179.

[3] K. Zhou, X. Wang, Y. Tong, M. Desbrun, B. N. Guo and H. Y. Shum, "Texture montage seamless texturing of arbitrary surfaces from multiple images", ACM Transactions on Graphics, vol.24, no.3, (2005), pp.1148-1155

[4] D. L. James, C. D. Twigg, "Skinning mesh animations", ACM Transactions on Graphics, vol.24, no.3, (2005), pp.399-407.

[5] E. Zuckerberger, A. Tal, S Shlafman, "Polyhedral surface decomposition with applications", Computers \& Graphics, vol.26. no.5, ( 2002), pp.733-743.

[6] H. Yamauchi, S. Y. Lee, Y. Lee, Y. Ohtake, A. Belyaev and H. P. Seidel, "Feature sensitive mesh segmentation with mean shift", Proceedings of International Conference on Shape Modeling and Applications, Washington DC, USA, (2005), pp. 238-245.

[7] C. Rossl, L. Kobbelt, H. P. Seidel. "Extract ion of feature lines on triangulated surfaces using morphological operators", Proceedings of the AAAI Symposium on Smart Graphics, Stanford, USA, (2001), pp. 71-75

[8] B. Hyoungseok, H. Kim, "Mesh segmentation based on local geometric properties", International Journal of Computer Science and Network Security, vol.10, no.1, (2010), pp. 179-184.

[9] Y. Xiao, P. Siebert, N. Werghi, "A discrete Reeb graph approach for the segmentation of human body scans", Proceedings of the 4th International Conference on 3D Digital Imaging and Modeling, Silver Spring, USA, (2003), pp.378-385.

[10] X. P. Sun, Q. Zhang, "3D Skeleton algorithm using level-progressive", Computer Science, vol.37, no.12, (2012), pp. 238-241.

[11] [11] A. Mangan, R. Whitaker, "Partitioning 3D surface meshes using watershed segmentation", IEEE Transactions on Visualization and Computer Graphics, vol.5, no.4, (1999), pp. 308-321.

[12] D. L. Page, A. F. Koschan, M. A. Abidi, "Perception based 3D triangle mesh segmentation using fast marching watersheds", Proceedings of Computer Vision and Pattern Recognition, Madison, USA, ( 2003), pp.27-32.

[13] Y. Zhang, J. K. Paik, M. A. Abidi, "A simple and efficient algorithm for part decomposition of 3D triangulated models based on curvature analysis", IEEE International Conference on Image Processing, Rochester, USA, (2002), pp.273-276.

[14] R. Liu, H. Zhang, "Segmentation of 3D meshes through spectral clustering", Proceeding of Pacific Graphics 2004, Washington DC, USA, (2004), pp. 298-305.

[15] H. Zhang, O. V. Kaick, R. Dyer, "Spectral methods for mesh processing and analysis", Computer Graphics Forum, vol.29, no.6, (2010), pp. 1865-1894.

[16] Y. Fisher, H. H. John, S. W. Ben, "A proof of the uniformization theorem for arbitrary plane domains", Proceedings of the American mathematical society, vol.104, no.2, (1998), pp. 413-418.

[17] M Ben-Chen, C Gotsman, "Characterizing shape using conformal factors", Proceedings on 3D Object Retrieval, Crete, Greece, (2008), pp.1-8.

[18] K. Hildebrandt, K. Polthier, M. Wardetzky, "On the convergence of metric and geometric properties of polyhedral surfaces", Geometria Dedicata, vol.123, no.1, (2006), pp. 89-112.

[19] J. Yang, Z. H. Tian, L. J. Li and X. P. Wang, "Segmentation of 3D geometric models based on mesh Laplace", Computer Science, vol.42, no.5, (2015), pp.295-299. 


\section{Authors}

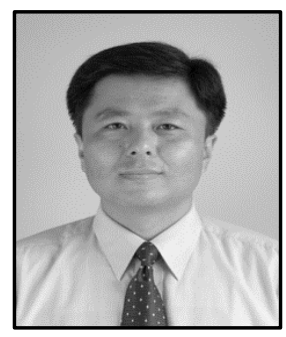

Yang Jun was born in 1973. He received the Ph.D. degree in computer science from Southwest Jiaotong University in 2007. Now he is a professor at School of Electronic and Information Engineering, Lanzhou Jiaotong University. His research interests include computer graphics, digital image processing, etc.

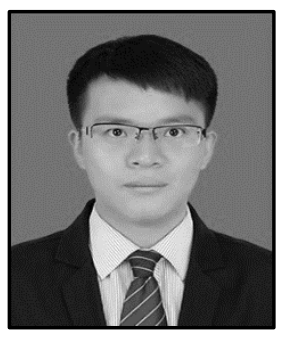

Wang Maozheng was born in 1987. He is a master candidate at School of Automation and Engineering, Lanzhou Jiaotong University. His research interests include control theory, control engineering, pattern recognition, etc.

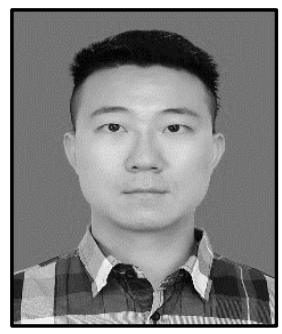

Yan Han was born in 1990. He is a master candidate at School of Electronic and Information Engineering, Lanzhou Jiaotong University. His research interests include computer graphics, pattern recognition, etc. 